\title{
Análisis de Composición Corporal y Somatotipo de Judokas Infantiles y Cadetes en el Campeonato de España 2012
}

\author{
Analysis of Body Composition and Somatotype of Junior \\ and Cadet Judokas in the 2012 Spanish Championship
}

Torres, F. J."; Lara-Padilla, E..*; Sosa-Tallei, G.*** \& Berral, F. J.****

TORRES, F. J.; LARA-PADILLA, E.; SOSA-TALLEI, G. \& BERRAL, F. J. Análisis de composición corporal y somatotipo de judokas infantiles y cadetes en el campeonato de España 2012. Int. J. Morphol., 32(1):194-201, 2014.

RESUMEN: El objetivo de este estudio es analizar la composición corporal y el somatotipo de judokas participantes en el

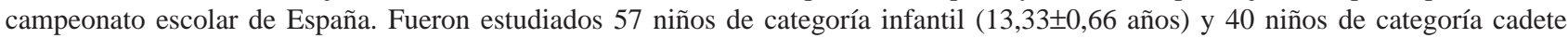
$(15,18 \pm 0,74$ años) (n=97), mediante un fraccionamiento corporal tetracompartimental y el análisis del somatotipo según el método de Heath-Carter. En categoría infantil obtuvimos una media de 48,11\% de masa muscular, 25,49\% de masa residual, 14,80\% de masa grasa y $11,56 \%$ de masa ósea. En categoría cadete, $47.67 \%$ de masa muscular, $25,25 \%$ de masa residual, $16,46 \%$ de masa grasa y $10,57 \%$ de masa ósea. Se encontraron diferencias significativas únicamente en el compartimiento de masa ósea entre ambos grupos. El somatotipo medio para ambas categorías es Ectomorfo Balanceado.

PALABRAS CLAVE: Judo; Antropometría; Fraccionamiento Tetracompartimental; Biotipo.

\section{INTRODUCCIÓN}

El Judo es un deporte caracterizado por complejas acciones motoras y capacidades motrices (Mansilla et al., 2000). Para alcanzar el máximo rendimiento, los judokas se someten a altas cargas de entrenamiento tanto a nivel técnico, táctico, psicológico y biológico (Boguszewski \& Boguszewska, 2006).

Con el transcurso de los años, se ha visto que en el Judo como en otras modalidades deportivas, cada vez es más importante que los deportistas adquieran unas características físicas determinadas, con el único objetivo de ser más competitivos. Por esto, en las disciplinas deportivas de combate, el rendimiento dependerá en gran medida de características antropométricas, como la composición corporal del deportista (Iide et al., 2008). Además, este deporte, cuenta con el factor del contacto corporal directo y continuo del adversario, así como la incertidumbre externa del mismo (Hernández \& Torres, 2007). Es por ello, por lo que la participación en un deporte concreto, debe estar asociada con los tres pilares de la Antropometría (Duquet \& Carter, 1996).
Según Mansilla (1999) los deportistas de alto nivel poseen un somatotipo, composición corporal y perfiles de proporcionalidad específicos, que nos llevan a relacionar el éxito en un determinado deporte con un prototipo físico definido y no con otro, sin olvidar los factores que intervienen en el rendimiento deportivo.

De igual manera, Dantas \& Fernandes (2005) afirman que "el somatotipo es un indicador del alto rendimiento deportivo". De esta manera, el biotipo que vayan definiendo los judokas en categorías inferiores irá determinando su potencial y un claro progreso en categorías absolutas. Basado en el contexto reseñado en los párrafos anteriores, surge la presente investigación bajo el firme propósito de aportar a la comunidad científica nuevos datos sobre la composición corporal de judokas de alto nivel pertenecientes a categorías inferiores.

Por todo esto, se ha llevado a cabo un estudio descriptivo e inferencial de prevalencia, transversal y de carácter

* Máster en Actividad Física y Salud. Doctorando de la Universidad Pablo de Olavide, Sevilla, España.

** Doctor en Alto Rendimiento Deportivo. Departamento de Estudios de Posgrado e Investigación. Escuela Superior de Medicina, Instituto Politécnico Nacional, México.

*** Especialista en Medicina del Deporte. Decano de la Facultad de Educación, Universidad Juan Agustín Maza, Mendoza, Argentina.

**** Catedrático de Universidad, Responsable del Grupo de Investigación CTS-595, Universidad Pablo de Olavide, Sevilla, España. 
prospectivo con una asignación aleatoria de los sujetos, analizando las características antropométricas de los judokas infantiles y cadetes clasificados para competir en el Campeonato Escolar de Judo de España procedentes de las diferentes Comunidades Autónomas.

\section{SUJETOS Y MÉTODO}

En este estudio han participado un total de 97 judokas masculinos $(n=97)$ entre las categorías infantil $(13,33 \pm 0,66$ años) y cadete $(15,18 \pm 0,74$ años $)$, procedentes de hasta 10 selecciones autonómicas, clasificados para participar en los Campeonatos de Judo de España 2012. Las mediciones se realizaron en el transcurso del Campeonato teniendo en cuenta la declaración de Helsinky, respecto a los principios éticos de investigación en seres humanos, donde cada delegado técnico autorizó la participación de forma voluntaria de sus deportistas, firmando para ello un consentimiento informado. Se han tenido en cuenta las recomendaciones de la Sociedad Internacional para el Avance de la Cineantropometría (ISAK), sobre selección de medidas antropométricas, técnicas e instrumental de medición, teniendo presentes los tres principios éticos básicos en investigación humana (respeto, beneficencia y justicia). Así mismo, todas las medidas se tomaron en el lado derecho del cuerpo aunque no fuese el lado dominante del Judoka. Con ello se determinó la composición corporal en cuatro compartimientos (masa muscular, masa ósea, masa residual y masa grasa). Para establecer el somatotipo se utilizó el modelo propuesto por Heath \& Carter (Carter, 1975). Hemos usado una báscula SECA 780, estadiómetro (precisión de 1 $\mathrm{mm}$ ), plicómetro Harpender, cinta métrica metálica Lufkin y paquímetro Holtain.

Con lo anterior, se determinaron de forma individual las variables peso, estatura en tracción e inspiración profun- da, índice de masa corporal, pliegues cutáneos (tríceps, subescapular, bíceps, pectoral, supracrestal, supraespinal, abdominal, frontal del muslo y medial de la pierna), perímetros corporales (brazo relajado y contraido, antebrazo máximo, muñeca mínimo, abdominal mínimo, gluteo máximo, muslo medio, máximo de la pierna y mínimo del tobi1lo), diámetros pequeños (humeral, biestiloideo, femoral y bimaleolar), masa muscular (\% - Kg) obtenida con la fórmula de Poortman et al. (2005), masa grasa (\% - Kg) utilizando la fórmula de Slaughter et al. (1988), masa ósea (\% $\mathrm{Kg}$ ) por Martin (1991) y masa residual (\% - Kg) por la relación propuesta por Würch (1974). Del mismo modo obtuvimos el somatotipo, con sus respectivas coordenadasy su clasificación biotipológica o cualitativa (Carter).

Para el análisis descriptivo se registraron, de cada variable, el promedio y desviación estándar de cada deportista. Con respecto al análisis inferencial, se analizó si realmente existen diferencias significativas en determinadas variables entre las categorías infantil y cadete. Para ello, atendiendo a la normalidad de los datos, se han utilizado pruebas paramétricas (t-Student) así como pruebas no paramétricas (U de Mann-Whitney) para establecer esas diferencias entre categorías. Así mismo, el nivel de significación se estableció en $\mathrm{p} \leq 0,05$ para todas las pruebas estadísticas realizadas.

\section{RESULTADOS}

En la Figura 1, se muestra la relación de deportistas analizados, según la Comunidad Autónoma, que han participado en el presente estudio, quedando finalmente configurada la muestra en 57 judokas infantiles y 40 cadetes.

En la Tabla I, se muestran los valores obtenidos en relación a las dimensiones e IMC, reflejándose que en cada

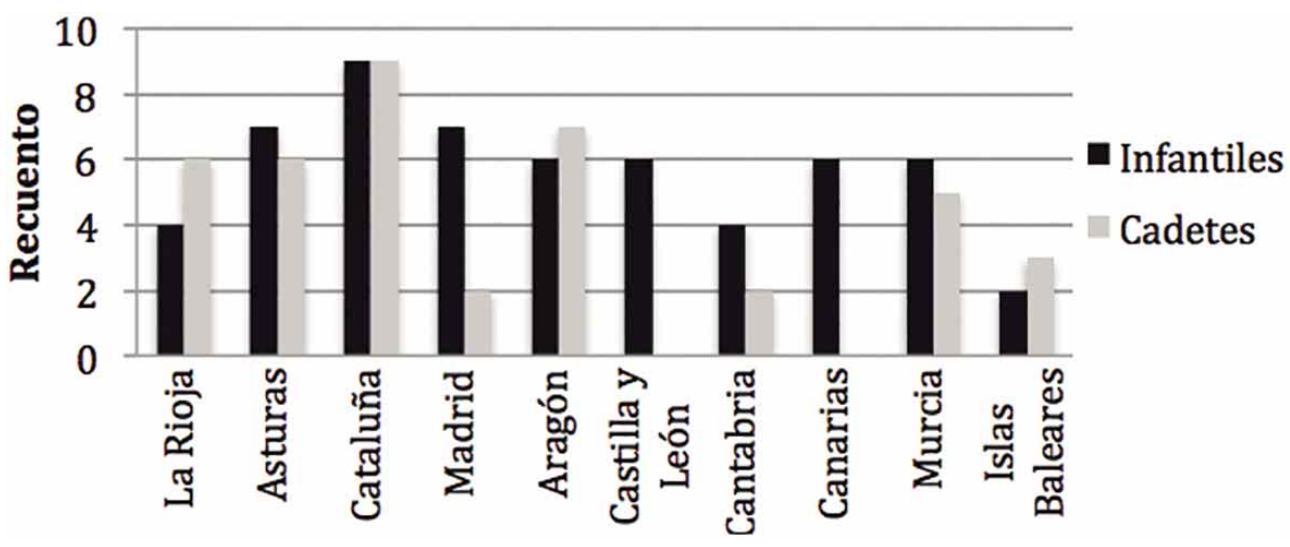

Fig. 1. Deportistas estudiados por Comunidades Autónomas. 
una de ellas existen diferencias estadísticamente significativas entre las categorías infantil y cadete.

En la Tabla II, podemos comprobar que los pliegues cutáneos, donde existen diferencias estadísticamente significativas entre los judokas infantiles y cadetes, son el abdominal $(0,017)$ y el subescapular $(0,017)$. En la Tabla III referente a perímetros corporales, observamos importantes di- ferencias entre las categorías infantil y cadete. Estas son significativas en todos los perímetros corporales, excepto en el perímetro mínimo del tobillo $(0,062)$.

En la Tabla IV, podemos ver que existen diferencias significativas entre las categorías infantil y cadete de judo en relación a los diámetros analizados, a excepción del diámetro óseo del tobillo o bimaleolar $(0,148)$, en el cual no se

Tabla I. Análisis inferencial del peso, estatura en bipedestación e IMC entre categorías Infantil (n= 57) Cadete $(n=40)$.

\begin{tabular}{lcccc}
\hline Variables e IMC & Medias \pm DE & Medias \pm DE & Sig. & Prueba Estadística \\
\hline Edad & $13,33 \pm 0,66$ & $15,18 \pm 0,74$ & & \\
Peso $(\mathrm{kg})$ & $51,96 \pm 10,26$ & $64,23 \pm 14,93$ & 0,000 & Mann - Whitney \\
Estatura $(\mathrm{cm})$ & $163,39 \pm 9,43$ & $170,73 \pm 7,69$ & 0,000 & t- Student \\
Índice Masa Corporal & $19,30 \pm 2,31$ & $21,80 \pm 3,40$ & 0,000 & Mann - Whitney \\
\hline
\end{tabular}

Tabla II. Análisis inferencial de pliegues cutáneos entre categorías.

\begin{tabular}{lcccc}
\hline Pliegues & Media \pm DE & Media \pm DE & Sig. & Prueba estadística \\
\hline Tríceps & $8,02 \pm 3,11$ & $9,03 \pm 4,39$ & 0,191 & t- Student \\
Bíceps & $4,80 \pm 1,70$ & $4,60 \pm 1,76$ & 0,280 & Mann - Whitney \\
Pectoral & $4,35 \pm 1,08$ & $4,46 \pm 1,28$ & 0,841 & Mann - Whitney \\
Abdominal & $8,56 \pm 4,70$ & $10,96 \pm 6,50$ & 0,017 & Mann - Whitney \\
Frontal Muslo & $11,71 \pm 4,58$ & $13,10 \pm 6,48$ & 0,526 & Mann - Whitney \\
Subesc apular & $7,35 \pm 2,54$ & $9,01 \pm 4,25$ & 0,017 & Mann - Whitney \\
Medial Pierna & $9,17 \pm 2,67$ & $9,75 \pm 4,02$ & 0,968 & Mann - Whitney \\
Supracrestal & $6,64 \pm 3,67$ & $8,10 \pm 5,61$ & 0,402 & Mann - Whitney \\
Supraespinal & $5,03 \pm 2,44$ & $6,07 \pm 3,99$ & 0,369 & Mann - Whitney \\
Sumatorio Pliegues Miembro Superior & $12,83 \pm 4,45$ & $13,63 \pm 6,00$ & 0,456 & t- Student \\
Sumatorio Pliegues del Tronco & $31,94 \pm 13,19$ & $38,61 \pm 20,54$ & 0,145 & Mann - Whitney \\
Sumatorio Pliegues Miembro Inferior & $20,89 \pm 6,98$ & $22,85 \pm 9,80$ & 0,600 & Mann - Whitney \\
\hline
\end{tabular}

Tabla III. Análisis inferencial de los perímetros corporales entre categorías.

\begin{tabular}{|c|c|c|c|c|}
\hline \multirow[b]{2}{*}{ Perímetros } & Infantil $(n=57)$ & Cadete $(n=40)$ & \multirow[b]{2}{*}{ Sig. } & \multirow[b]{2}{*}{ Prueba Estadística } \\
\hline & Medias $\pm \mathrm{DE}$ & Medias $\pm \mathrm{DE}$ & & \\
\hline Glúteo máximo & $81,94 \pm 8,84$ & $90,08 \pm 9,83$ & 0,000 & t- Student \\
\hline Pierna máximo & $33,20 \pm 3,69$ & $36,45 \pm 4,45$ & 0,001 & Mann - Whitney \\
\hline Muslo medio & $45,43 \pm 4,54$ & $50,06 \pm 5,87$ & 0,000 & Mann - Whitney \\
\hline Abdominal mínimo & $68,88 \pm 6,27$ & $74,25 \pm 9,14$ & 0,001 & Mann - Whitney \\
\hline Antebrazo máximo & $23,76 \pm 2,19$ & $25,97 \pm 2,39$ & 0,000 & t- Student \\
\hline Brazo relaja do & $25,07 \pm 2,77$ & $27,79 \pm 3,56$ & 0,000 & t- Student \\
\hline Muñeca mínimo & $16,15 \pm 0,94$ & $16,76 \pm 0,93$ & 0,002 & t- Student \\
\hline Tobillo mínimo & $21,96 \pm 1,57$ & $22,76 \pm 2,47$ & 0,062 & Mann - Whitney \\
\hline Brazo contraído & $27,98 \pm 2,95$ & $31,02 \pm 3,49$ & 0,000 & t- Student \\
\hline
\end{tabular}

Tabla IV. Análisis inferencia de los diámetros óseos entre categorías.

\begin{tabular}{|c|c|c|c|c|}
\hline \multirow[b]{2}{*}{ Diáme tros } & Infantil $(n=57)$ & Cadete $(n=40)$ & \multirow[b]{2}{*}{ Sig. } & \multirow[b]{2}{*}{ Prueba Estadística } \\
\hline & Medias \pm DE & Medias \pm DE & & \\
\hline Humeral & $5,40 \pm 0,53$ & $5,75 \pm 0,59$ & 0,003 & t- Student \\
\hline Biestiloideo & $4,39 \pm 0,34$ & $4,60 \pm 0,34$ & 0,006 & t- Student \\
\hline Femoral & $8,54 \pm 0,55$ & $8,81 \pm 0,75$ & 0,047 & t- Student \\
\hline Bimale olar & $5,61 \pm 0,54$ & $5,82 \pm 1,05$ & 0,148 & Mann - Whitney \\
\hline
\end{tabular}


TORRES, F. J.; LARA-PADILLA, E.; SOSA-TALLEI, G. \& BERRAL, F. J. Análisis de composición corporal y somatotipo de judokas infantiles y cadetes en el campeonato de España 2012. Int. J. Morphol., 32(1):194-201, 2014.

Tabla V. Análisis inferencial del fraccionamiento tetracompartimental entre categorías.

\begin{tabular}{lcccc}
\hline & Infantil $(\mathbf{n}=\mathbf{5 7})$ & Cadete $(\mathbf{n}=\mathbf{4 0})$ & & \\
\cline { 2 - 3 } $\begin{array}{l}\text { Fraccionamiento } \\
\text { Tetracompartimental }\end{array}$ & Medias \pm DE & Medias \pm DE & \multirow{2}{*}{ Sig. } & Prueba Estadística \\
\hline Masa Muscular (\%) & $45,63 \pm 2,53$ & $45,64 \pm 3,11$ & 0,994 & Mann - Whitney \\
Masa Residual (\%) & $24,10 \pm 0,00$ & $24,10 \pm 0,00$ & & \\
Masa Grasa (\%) & $13,64 \pm 3,94$ & $14,80 \pm 5,86$ & 0,613 & Mann - Whitney \\
Masa ósea (\%) & $11,04 \pm 1,32$ & $10,21 \pm 1,37$ & 0,003 & t- Student \\
\hline
\end{tabular}

Tabla VI. Análisis inferencial del somatotipo entre categorías.

\begin{tabular}{|c|c|c|c|c|}
\hline & Infantil $(n=57)$ & Cadete $(n=40)$ & & \\
\hline Somatotipo & Medias $\pm \mathrm{DE}$ & Medias $\pm \mathrm{DE}$ & Sig. & Prueba estadística \\
\hline Endo & $2,21 \pm 0,95$ & $2,50 \pm 1,27$ & 0,429 & Mann - Whitney \\
\hline Meso & $2,63 \pm 1,22$ & $3,13 \pm 1,30$ & 0,053 & t- Student \\
\hline Ecto & $3,63 \pm 1,15$ & $2,87 \pm 1,34$ & 0,003 & t- Student \\
\hline
\end{tabular}

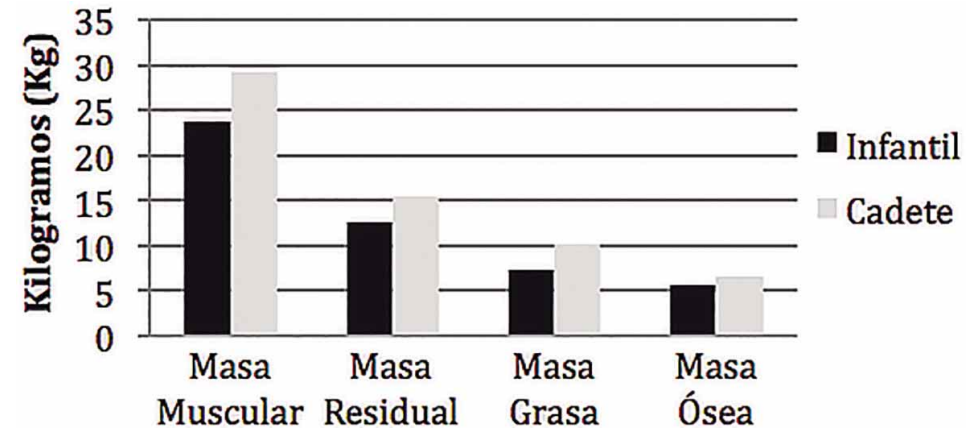

Fig. 2. Comparación de la composición corporal en Kg por compartimentos entre categorías Infantil y Cadete.

muestran diferencias. Tanto en la Tabla V como en la Figura 2, se observa la existencia de diferencias significativas en relación al fraccionamiento tetracompartimental de la composición corporal entre las categorías infantil y cadete.

En la Figura 3, se muestran las frecuencias somatotípicas en ambas categorías, reflejándose que el somatotipo más común es Ectomorfo Balanceado, tanto en categoría infantil como cadete. En la Tabla VI, observamos como en el componente somatotípico de la Ectomorfia, se encuentran diferencias significativas $(0,003)$ entre los judokas infantiles y cadetes. Así mismo en la Figura 4, se muestra la Somatocarta con el somatotipo poblacional de los deportistas.

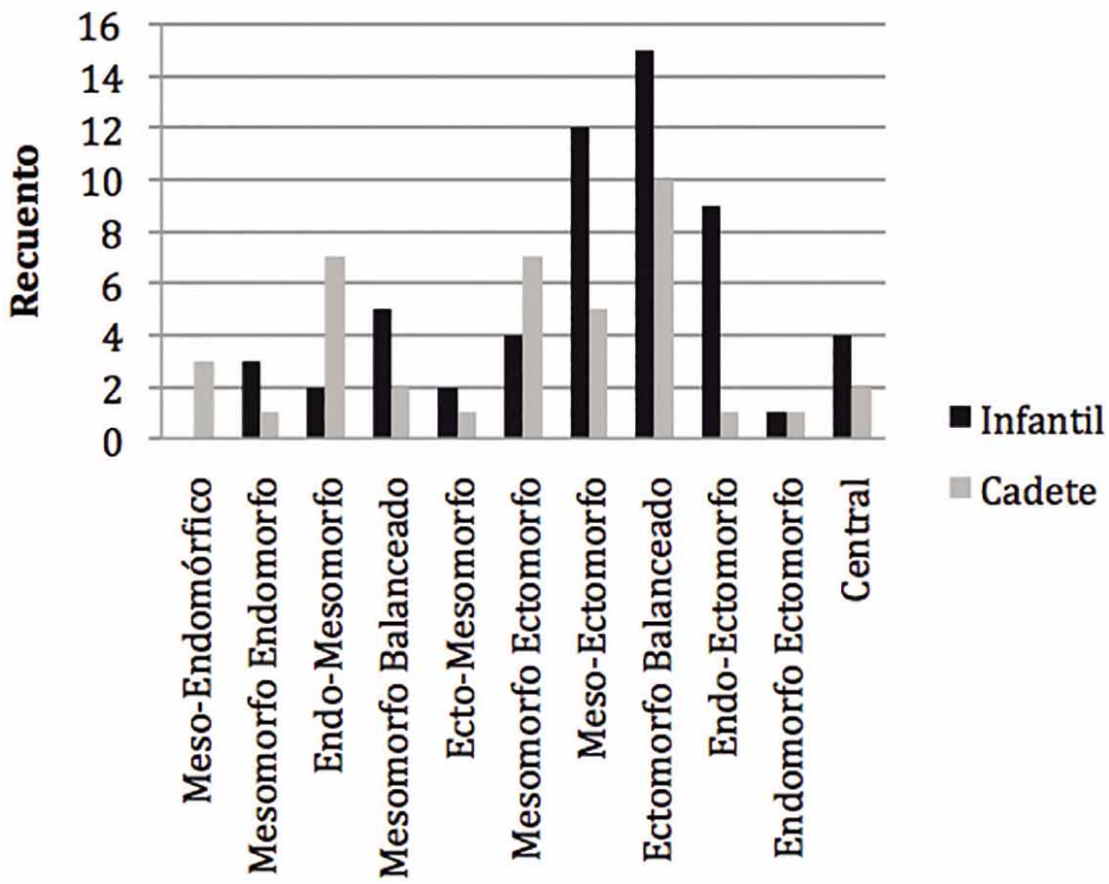

Fig. 3. Comparación del somatotipo entre las categorías infantil y cadete. 


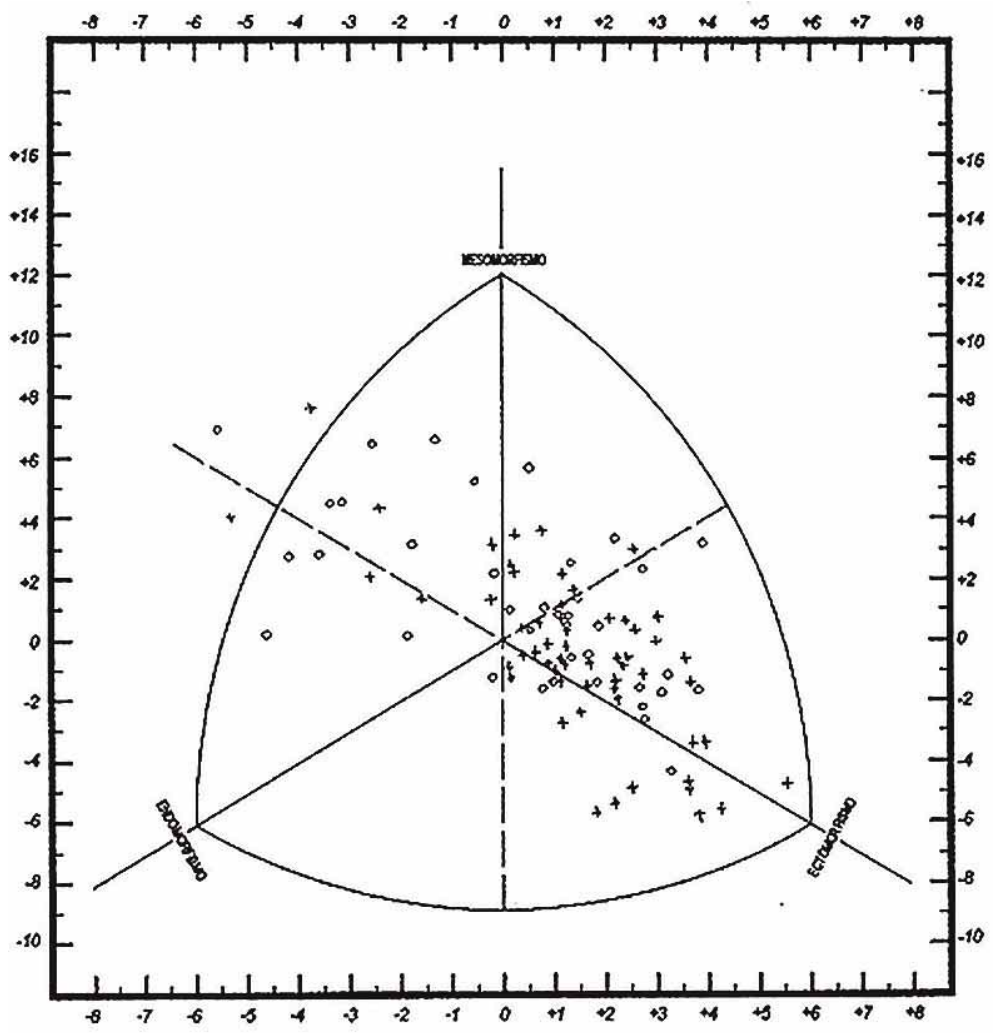

taekwondo el somatotipo promedio obtenido mediante el método Heath y Carter, es MesoEctomórfico, mientras que en judo, lucha libre, lucha grecorromana fue MesoEndomórfico. Estos datos nos indican la variabilidad biotipológica en el deporte de alto nivel. Sin embargo y centrándonos en el somatotipo de los judokas, en nuestro estudio en categorías de formación, se han observado biotipos medios bien diferentes respecto al alto nivel, con un biotipo Ectomorfo Balanceado para ambas categorías, lo que nos indica de los importantes cambios que han de experimentar la categorías en formación, hasta llegar a categorías absolutas.

Rodríguez \& Berral (2006) realizan un estudio de composición corporal por medio del método de Kerry del somatotipo con el método antropométrico Heath-Carter, en gimnastas argentinos de alto rendimiento de categorías cadete, junior y séniors, encontrando valores muy diferentes en todos los compartimientos, masa muscular $53,77 \%$, masa grasa $17,48 \%$, masa ósea $11,74 \%$; masa residual $11,85 \%$, con respecto a los judokas infantiles y cadetes del

Fig. 4. Somatocarta de las categorías infantil y cadete. $+=$ Infantiles; $\mathrm{O}=$ Cadetes.

\section{DISCUSIÓN}

Son escasos los estudios que tratan de analizar la composición corporal y el somatotipo en el deporte del Judo, y aún menos en categorías de formación. Sin embargo, en otros deportes o incluso para poblaciones geográficas determinadas, sí que han tenido cabida una gran cantidad de estudios en esta línea de investigación. Berral et al. (2001) realizan un estudio de la composición corporal en escolares entre 10 a 14 años utilizando las fórmulas de Lohman \& Slaughter, observando valores de pliegues cutáneos muy similares a los obtenidos en este estudio para ambas categorías, en los que obtuvimos una media sumatoria de $12,83 \mathrm{~mm}$ en el miembro superior con respecto a $20,89 \mathrm{~mm}$ en el miembro inferior en categoría Infantil, así como 13,63 mm en el miembro superior y $22,85 \mathrm{~mm}$ en el miembro inferior en categoría Cadete, en relación a los $20 \mathrm{~mm}$ en el miembro superior y $33 \mathrm{~mm}$ en el miembro inferior del trabajo referenciado.

Por otro lado, en un estudio antropométrico de Betancourt et al. (2009), en deportistas de élite Cubanos en la modalidad de combate, encuentran como en el boxeo y presente estudio (Tabla V), por otro lado lógico al haber sido obtenidas estas masas por metodologías diferentes no comparables. Pero si comparamos los valores de distintas variables, observamos como los judokas de nuestro estudio (Tabla III) presentan menores dimensiones en todos perímetros con respecto a los gimnastas argentinos: brazo relajado $(32,2 \mathrm{~cm})$, brazo flexionado y contraído ( $35 \mathrm{~cm})$, antebrazo máximo ( 28 $\mathrm{cm})$, muñeca mínimo $(17,9 \mathrm{~cm})$, abdomen mínimo $(76,7 \mathrm{~cm})$, glúteo máximo $(89,6 \mathrm{~cm})$, muslo medio $(53,1 \mathrm{~cm})$ y máximo de la pierna $(36,1 \mathrm{~cm})$. De igual modo, los diámetros óseos también resultan ser mayores en los gimnastas argentinos con respecto a los judokas infantiles y cadetes (Tabla IV) siendo el humeral $(7,1 \mathrm{~cm})$, biestiloideo $(6,1 \mathrm{~cm})$ y femoral $(9,5$ $\mathrm{cm})$. La media obtenida del somatotipo de los gimnastas argentinos es 1,9-6,7-1,6, teniendo por tanto unos índices menores en los componentes de la Endomorfia y Ectomorfia, así como valores muy superiores en la Mesomorfia con respecto a los judokas de este estudio.

Berral et al. (2003), en un estudio morfológico realizado a ciclistas de 17 y 18 años participantes en competiciones nacionales de España, determina la composición corporal utilizando el método penta compatimerntal de Kerr, obteniendo unos porcentajes parecidos de masa muscular $(45,61 \%)$ y masa ósea $(12,23 \%)$ respecto a los judokas analizados en nuestro trabajo (Tabla V) a pesar de utilizar metodología diferente, diferenciándose eso sí en gran medida 
en los compartimentos de masa grasa $(23,95 \%)$ y la masa residual $(12,06 \%)$. Señalar que la grasa obtenida por el método Kerr corresponde a la grasa total (esencial y corporal de reserva).

Atendiendo a las variables antropométricas generales, comprobamos como los judokas de categoría cadete, muestran un mayor peso $(64,23 \mathrm{~kg})$ que los ciclistas de $17 \mathrm{y}$ 18 años $(63,23 \mathrm{~kg})$ a pesar de tener una menor estatura, indicándonos las características requeridas en ambos deportes para alcanzar un mayor rendimiento. Así mismo en el somatotipo medio de los ciclistas Ecto-Mesomorfos, se aprecian ciertas diferencias respecto al biotipo Ectomorfo Balanceado de los judokas.

Posteriormente Berral et al. (2010), en un estudio donde analizan la masa muscular en jugadores junior de Bádminton, encuentran resultados ligeramente inferiores en sus porcentajes de masa muscular utilizando la fórmula de Drinkwater (1984) (40,07\% con respecto al $45 \%$ de los judokas) y masa ósea por medio de la fórmula de Von Döbeln (1964) modificada por Rocha (1975) (17\% con respecto a los $10,2 \%$ de los judokas). Con respecto a los otros compartimientos, apenas se aprecian diferencias con valores muy similares tanto en la masa residual (24.1\%) como en la masa grasa $(13,27 \%)$. Al valorar las variables antropométricas de forma independiente observamos como en los perímetros corporales, los jugadores de bádminton mostraron menores dimensiones en brazo relajado $(27,06 \mathrm{~cm})$, antebrazo máximo $(25,55 \mathrm{~cm})$ y máximo de la pierna $(36 \mathrm{~cm})$, así como un mayor perímetro en el muslo medio $(56,26 \mathrm{~cm})$. Sin embargo, los diámetros óseos resultaron mayores en los jugadores de bádminton, tanto en el biestiloideo $(5,50 \mathrm{~cm})$ como en el femoral $(9,41 \mathrm{~cm})$.

En el mismo año, Gómez-Landero et al. (2010), en un estudio donde se analiza la composición corporal y el somatotipo mediante el método de Heath-Carter en gimnastas de trampolín en categoría cadete, los resultados encontrados indican que estos saltadores tienen un mayor predominio en el componente de la Mesomorfia $(4,98)$ con respecto a los judokas de este estudio (Tabla VI), mientras que en el resto de componentes no difieren de manera importante (Endo 2,75; Ecto 2,76). Además estos saltadores manifiestan un mayor porcentaje de masa ósea obtenido por la fórmula de Von Doblen modificada por Rocha de 19,15\% y menor masa grasa por la fórmulade Faulkner (1968) de $10,38 \%$ debido a que este no utiliza pliegues del miembro inferior en la estimación de este compartimiento. La masa muscular en los gimnastas de trampolín, fue de 46,38\%, obteniéndose por tanto valores muy parecidos en la masa muscular de los judokas infantiles $(45,63 \%)$ y cadetes $(45,64 \%)$ de nuestro estudio.
Analizando las variables antropométricas por separado, observamos como los gimnastas de trampolín, muestran un menor peso $(49,91 \mathrm{~kg})$, estatura $(155,87 \mathrm{~m})$ e IMC $(19,96)$ que los judokas de categoría infantil (Tabla I).

Hernández et al. (2009), en un estudio donde analizan la variabilidad de los parámetros antropométricos en judokas de alto rendimiento con una edad media de 20,7 años, pertenecientes a la Selección Nacional, reflejan unos valores en la composición corporal, utilizando la táctica de De Rose \& Guimaraes, en el compartimento de masa grasa de $16,5 \%$, porcentaje mayor que el obtenido en los judokas infantiles $(13,64 \%)$ y cadetes $(14,80 \%)$ de este estudio, aunque por distintos métodos, no pudiendose llegar a conclusiones entre ambas muestras en relación a este compartimiento.

Torres-Luque et al. (2011), analizan las variaciones antropométricas de judokas de alto nivel con una media de edad de 21 años, tras un periodo de entrenamiento de 7 semanas y con ello comprueban cómo se modifica el porcentaje en sus compartimientos corporales por medio de la táctica de Rose \& Guimares.

Carratalá et al. (2004), en su estudio pretendían determinar el perfil antropométrico de jóvenes luchadoras pertenecientes a la selección española de judo de las categorías infantil y cadete. A pesar de pertenecer a la misma categoría de edad, son de distinto sexo, por lo que esta comparación será únicamente descriptiva. Sus resultados en la composición corporal fueron de un 20,83\% de masa grasa, $16,55 \%$ de masa ósea, así como un $41,72 \%$ de masa muscular en la categoría infantil. Con respecto a la categoría cadete, se obtuvieron unos porcentajes de 19,93\% de masa grasa, $16,58 \%$ de masa ósea y un $42,57 \%$ de masa muscular, no describiendo para ambos grupos el método utilizado para su cálculo. Los resultados obtenidos en el somatotipo femenino medio mediante el método Heath-Carter nos indican que para la categoría infantil es de Mesomorfo-Endomorfo, mientras que en cadete es Endo-Mesomorfo, ambos diferenciándose en gran medida con respecto a la linealidad (Ectomorfo balanceado) de los sujetos varones de este estudio. Al valorar las variables antropométricas de forma independiente observamos que los judokas cadetes de nuestro estudio presentan unos perímetros del brazo contraído $(31,02 \mathrm{~cm})$ y antebrazo máximo $(25,97 \mathrm{~cm})$ mayor que las judokas cadetes $(28,35 \mathrm{~cm}$ y $23,52 \mathrm{~cm}$ respectivamente) del estudio realizado por Carratalá et al. Sin embargo, los perímetros del muslo medio y pierna máximo en los judokas infantiles $(45,43 \mathrm{~cm})$ y cadetes $(50,06 \mathrm{~cm})$ de nuestro estudio, son menores que los perímetros de las judokas infantiles $(52,85$ $\mathrm{cm})$ y cadetes $(52,67 \mathrm{~cm})$ del estudio referenciado. Por otro lado, las judokas infantiles y cadetes de este estudio tam- 
bién muestran un diámetro óseo biestiloideo mayor $(4,86$ $\mathrm{cm}$ infantiles y $5,11 \mathrm{~cm}$ cadetes) que los judokas masculinos de nuestro estudio. Por último, las judokas tanto infantiles como cadetes, muestran un mayor espesor en la mayoría de los pliegues cutáneos (tríceps, subescapular, bicipital, supraespinal, abdominal, frontal del muslo y medial de la pierna) con respecto a los judokas masculinos de nuestro estudio, indicándonos claramente las diferencias existentes entre sexos.

Flores (2009) en su Tesis Doctoral determina el perfil antropométrico de la Selección Peruana de Judo en las categorías infantil, juvenil, junior y mayores. Los resultados del somatotipo medio en la categoría infantil fue MesoEndomórfico con unos porcentajes en la composición corporal por medio de la propuesta de Ross \& Kerr (1993) muy elevados de masa grasa $(27,3 \%)$, similares en la masa ósea $(12.1 \%)$, ligeramente inferiores en la masa muscular $(42,6 \%)$, y claramente inferiores en relación a la masa residual $(11,5 \%)$, habiéndose obtenidas estas masas por metodologías diferentes a la nuestra y no comparables. En este estudio no se detallan valores de variables antropométricas que podamos comparar. El somatotipo Meso-Endomórficode los judokas infantiles peruanos se diferencia sustancialmente de los judokas infantiles españoles, indicándonos el mayor porcentaje graso en la muestra peruana. En la categoría juvenil, que es la más próxima a la cadete, se determinó un somatotipo medio Mesomórfico Balanceado, con los siguientes componen- tes corporales: masa grasa $25,1 \%$, masa muscular $47,5 \%$, masa ósea $9 \%$ y masa residual $11,9 \%$ y por tanto, estos resultados difieren mucho con nuestro estudio en el porcentaje de la masa grasa y el somatotipo.

Torres-Luque et al. (2008), estudian las características físicas, así como otras pruebas de condición física del judoka adolescente, participantes en el Campeonato de España de 2008. En relación a la composición corporal, estiman el porcentaje graso mediante un método de laboratorio, la Impedancia Bioeléctrica Doble (TANITA BC 532), obteniendo un porcentaje graso por este método, no comparable al nuestro, de $12,67 \%$, valor muy próximo a los obtenidos en nuestro trabajo de $13,64 \%$ en categoría infantil y de $14,80 \%$ en categoría cadete.

También Úbeda et al. (2010), estudian los hábitos alimenticios y la composición corporal de deportistas españoles de élite en deportes de combate, utilizando el método de laboratorio de Absorciometría dual fotónica de rayos X (DEXA), mediante el que obtienen un valor de masa grasa del $10,5 \%$, no comparable a nuestro estudio.

\section{AGRADECIMIENTOS}

A las Federaciones Autonómicas participantes por su colaboración desinteresada.

TORRES, F. J.; LARA-PADILLA, E.; SOSA-TALLEI, G. \& BERRAL, F. J. Analysis of body composition and somatotype of junior and cadet judokas in the 2012 spanish championship. Int. J. Morphol., 32(1):194-201, 2014.

SUMMARY: The aim of the study was to analyze body composition and somatotype of the judokas participants at the Spanish Junior Championship. We analyzed 97 male judokas $(n=97)$ : 57 junior $(13.33 \pm 0.66$ years $)$ and 40 cadets $(15.18 \pm 0.74$ years $)$, through a Tetracompartimental Fractionation and the somatotype through a Heath-Carter method. In junior category, $48.11 \%$ of muscle mass, $25.49 \%$ of residual mass, $14.80 \%$ of fat mass and $11.56 \%$ of bone mass. In cadet category, $47.67 \%$ of muscle mass, $25.25 \%$ of residual mass, $16.46 \%$ of fat mass and $10.57 \%$ of bone mass. Significant differences were found only in the bone compartment between both groups. The average somatotype for both categories was Ectomorph Balanced.

KEY WORDS: Judoka; Tetracompartimental Fractionation; Somatotype; Body Composition; Anthropometry.

\section{REFERENCIAS BIBLIOGRÁFICAS}

Berral, F. J.; Gómez, J.; Hernán, B.; Berral, C. J. \& Carpintero, P. Estudio de la composición corporal en escolares de 10 a 14 años. Rev. Bras. Cineantropom. Desempenho Hum., 3(1):2033, 2001.

Berral, F. J.; Salinas, L.; Berral, C. J.; Lima, M. C. \& Carpintero, P. Estudio morfológico y funcional de ciclistas federados. Rev. Antioq. Med. Deportiva, 6(1):19-29, 2003.
Berral, F. J.; Rodríguez-Bies, E. C.; Berral, C. J.; Rojano, D. \& Lara, E. Comparación de ecuaciones antropométricas para evaluar la masa muscular en jugadores de bádminton. Int. J. Morphol., 28(3):803-10, 2010.

Betancourt, H.; Aréchiga, J. \& Carvajal, W. Estimación antropológica de la forma corporal de atletas elites cubanos de deportes olímpicos de combate. Antropol., 19:23-32, 2009. 
Boguszewski, D. \& Boguszewska, K. Dynamics of judo contests performed by finalists of European Championships (Rotterdam 2005). Arch. Budo, 2:40-4, 2006.

Carratalá, E.; Benavent, J. \& Carqués, L. Valoración de los componentes cineantropométricos de los judokas infantiles y cadetes del equipo nacional español. Universitat de Valencia. III Congreso de la Asociación Española de Ciencias del Deporte, Valencia, España, 2004.

Carter, J. The Heath-Carter Somatotype Method. San Diego, San Diego State University, 1975.

Dantas, E. \& Fernandes, J. Atividade Física em Ciencias da Saúde. Rio de Janeiro, Shape, 2005.

Drinkwater, D. T. An anatomically derived method for the anthropometric estimation of human body composition. $\mathrm{PhD}$ Thesis, Simon Fraser University, 1984.

Duquet, W. \& Carter, J. Somatotyping. En: Eston, R. \& Reilly, T. (Eds.). Kinanthropometry and exercise physiology laboratory manual. Londres, E \& FN Spon, 1996. pp.35-50.

Faulkner, J. A. Physiology of swimming and diving. En: H. Falls (Ed.). Exercise Physiology. Baltimore, Academic Press, 1968.

Flores, R. I. J. A. Perfil cineantropométrico de la selección peruana de judo infantil, juvenil, junior, mayores 2009. Tesis Doctoral, Lima, Universidad Nacional Mayor de San Marcos, 2009.

Gómez-Landero, L.; Vernetta, M. \& López, J. Somatotipo y composición corporal en gimnastas de Trampolín masculino español de alto nivel. Rev. Int. Cienc. Deporte, 6(19):141-53, 2010.

Hernández, G. R. \& Torres, G. Análisis temporal del combate de judo en competición. Rev. Int. Med. Cienc. Act. Fís. Deporte, 25:52-60, 2007.

Hernández, G. R.; Torres, L. G. \& Escobar, M. R. Evolución Antropométrica y electrolítica en un periodo competitivo en judokas de élite. $C C D, 5(5): 103-10,2009$.

Iide, K.; Imamura, H.; Yoshimura, Y.; Yamashita, A.; Miyahara, K.; Miyamoto, N. \& Moriwaki, C. Physiological responses of simulated karate sparring matches in young men and boys. $J$. Strength Cond. Res., 22(3):839-44, 2008.

Mansilla, M. Perfil funcional del competidor de lucha leonesa: comparación con otros deportes de lucha, el judo. Tesis Doctoral, León, Universidad de León, 1999.

Mansilla, M.; Villa, J.; García, J. \& López, C. Comparación de diferentes manifestaciones de fuerza y flexibilidad entre luchadores de lucha leonesa y judokas. I Congreso Nacional de la Asociación Española de Ciencias del Deporte, Cáceres, España, 2000.

Martin, A. Anthropometric assessment of bone mineral. En: Himes, J. (Ed.). Anthropometric assessment of nutritional status. New York, Wiley-Liss, 1991. pp.185-96.
Poortmans, J.; Boisseau, N.; Moraine, J.; Moreno-Reyes, R. \& Goldman S. Estimation of total body skeletal muscle mass in children and adolescents. Med. Sci. Sports Exerc., 37(2):31622, 2005.

Rocha, M. S. L. Peso óseo do brasileiro de ambos os sexos de 17 a 25 anhos. Arq. Anat. Antrop., 1:445-51, 1975.

Rodríguez, B. E. \& Berral, R. F. J. Estudio morfológico en gimnastas argentinos de alto rendimiento. Rev. Bras. Cineantropom. Desempenho Hum., 8(4):16-24, 2006.

Ross, W. D. \& Kerr, D. A. Fraccionamiento de la masa corporal: un nuevo método para utilizar en nutrición clínica y medicina deportiva. Apunts, Educ. Fís. Deport., 18:175-87, 1993.

Slaughter, M. H.; Lohman, T. G.; Boileau, R. A.; Horswill, C. A.; Stillman, R. J.; Van Loan, M. D. \& Bemben, D. A. Skinfold equations for estimation of body fatness in children and youth. Hum. Biol., 60(5):709-23, 1988.

Torres-Luque, G.; Hernández-García, R. \& Orquín, F. Características físicas del Judoka masculino adolescente. IV Congreso Internacional y XXV Nacional de Educación Física, Córdoba, España, 2008.

Torres-Luque, G.; Hernández-García, R. \& Garatachea, V. N. Variaciones antropométricas a lo largo de un periodo competitivo en judokas de élite. Rev. Int. Cienc. Deporte, VII(24):203-15, 2011.

Úbeda, N.; Palacios, G-A. N.; Montalvo, Z. Z.; García, J. B.; García, Á. \& Iglesias-Gutiérrez, E. Hábitos alimenticios y composición corporal de deportistas españoles de élite pertenecientes a disciplinas de combate. Nutr. Hosp., 25(3):414-21, 2010.

Von Döbeln, W. Determination of body constituents. En: Blix, G. (Ed.). Occurrences, causes and prevention of overnutrition. Upsala, Almquist and Wiksell, 1964.

Würch, A. La femme et le sport. Med. Sport. Française, 4(1): 441445, 1974.

Dirección para Correspondencia:

Francisco Jose Berral de la Rosa

Departamento de Deporte e Informática

Universidad Pablo de Olavide

Carretera de Utrera Km 1. 41013

Sevilla

ESPAÑA

Email: fjberde@upo.es

Recibido : 22-11-2012

Aceptado: 24-12-2013 